76 Diagnosis and management of secretory otitis media

A Varying head posture may alter the degree of deafness.

B Pain is an uncommon feature.

C A mobile eardrum excludes the diagnosis.

D Cortical mastoidectomy may be necessary.

E Chronic suppurative otitis media with cholesteatoma may be a late sequela.

\title{
77 Late sequelae of otitis media
}
A Middle ear atelectasis is usually sited in the posterior half of the ear drum and may be reversed by ventilation.
B Adhesive otitis media can be satisfactorily managed by division of fibrous bands and insertion of silastic sheeting.
C The commonest site of intratympanic tympanosclerosis is the stapedius tendon.
D Dry perforations of the pars flaccida are present in about $60 \%$ of cases of middle ear tympanosclerosis.
E Attic tympanosclerosis may result in the 'fixed malleus syndrome'.

\section{Tuberculous otitis media is}
A Usually painless.
B Associated with multiple perforations of the pars tensa.
C Occasionally heralded by a mastoid complication such as facial paralysis.
D Possibly contracted by aspiration of milk via the eustachian tube.
E Caused by treponema pallidum. 